\title{
Efficient Killing of Multidrug-Resistant Intracellular Bacteria by AlEgens in Vivo
}

Ying Li ${ }^{\left[{ }^{[*]}\right.}$ Fei Liu, ${ }^{[\dagger]}$ Jiangjiang Zhang, Xiaoye Liu, Peihong Xiao, Haotian Bai, Shang Chen, Dong Wang, Simon H. P. Sung, Ryan T. K. Kwok, Kui Zhu,*Ben Zhong Tang*

Dr. Y. Li, Dr. P. H. Xiao, Dr. D. Wang, Prof. B. Z. Tang

Center for AIE Research, College of Materials Science and Engineering, Shenzhen

University, Shenzhen 518060, China

F. Liu, Dr. X. Y. Liu, S. Chen, Prof. K. Zhu

Beijing Advanced Innovation Center for Food Nutrition and Human Health, College of Veterinary Medicine, China Agricultural University, No. 2 Yuanmingyuan West Rd, Beijing 100193, China

E-mail: zhuk@cau.edu.cn

Dr. J. J. Zhang

Department of Biomedical Engineering, Southern University of Science and Technology, No. 1088 Xueyuan Rd, Nanshan District, Shenzhen, 518055, China

Dr. H. T. Bai, Dr. S. H. P. Sung, Dr. R. T. K. Kwok, Prof. B. Z. Tang

Department of Chemistry, Hong Kong Branch of Chinese National Engineering Research Center for Tissue Restoration and Reconstruction, Institute for Advanced Study, Division of Life Science, The Hong Kong University of Science and Technology, Clear Water Bay, Kowloon, Hong Kong, China

E-mail: tangbenz@ust.hk

${ }^{[+]}$These authors contributed equally to this work.

Keywords: aggregation-induced emission luminogen, antibiotic, intracellular bacteria, autophagy, MRSA

Bacteria infected cells acting as "Trojan horses" not only protect bacteria from antibiotic therapies and immune clearance, but also increase the dissemination of pathogens from the initial sites of infection. Antibiotics are hard and insufficient to treat such hidden intracellular bacteria, especially the multidrug-resistant (MDR) bacteria. Herein, aggregation-induced emission luminogens (AIEgens) such as TBPs showed potent broad-spectrum bactericidal activity against both extracellular and intracellular Gram-positive pathogens at low-dose levels. TBPs triggered reactive oxygen species (ROS)-mediated membrane damage to kill bacteria, 
regardless of light irradiation. Additionally, such AIEgens activated mitochondria dependent autophagy to eliminate intracellular bacteria in host cells. Compared to the routinely used vancomycin in clinics, TBPs showed comparable efficacy against methicillin-resistant Staphylococcus aureus (MRSA) in vivo. Our studies demonstrate that AIEgens are promising new agents for the treatment of MDR bacteria associated infections.

\section{Introduction}

Pathogenic bacteria pose severe illness and significant mortality globally. The discovery of penicillin in 1928 and its subsequent introduction to clinic is a cornerstone of modern medical system. Since then, numerous classes of antibiotics have saved countless individuals from bacterial infections. However, such ability to cure has been dramatically impaired due to the emergence and dissemination of antibiotic resistance. ${ }^{[1]}$ Infections associated with multidrugresistant (MDR) bacteria, such as methicillin-resistant Staphylococcus aureus (MRSA) and vancomycin-resistant enterococci (VRE), are increasing to overwhelm nosocomial treatments worldwide, leading to high mortalities. ${ }^{[2-4]}$ The Centers for Disease Control (CDC) and Prevention estimate that more than two million people suffer from antibiotic resistant infections and at least 23000 people die each year in the United States alone. ${ }^{[5]}$ The rising rates of MDR bacterial pathogens constitute a serious threat to people health. ${ }^{[6]}$ Additionally, infections with MDR bacteria are notorious to treat due to the invasion and survival of them in mammalian cells, exhibiting similar behaviors to typical intracellular bacteria. For example, $S$. aureus $^{[7]}$ and Klebsiella pneumoniae ${ }^{[8]}$ are able to survive inside mammalian cells. Bacteria infected cells act as "Trojan horses", which not only protect bacteria from antibiotic treatments and immune clearance, but also may increase the dissemination of bacteria from the initial infection sites. Conventional antibiotics are difficult to kill such intracellular bacteria. Therefore, it is urgent to develop alternative strategies and antibiotics, for the better treatment of MDR bacterial pathogens. 
Antibacterial mechanisms of clinically available antibiotics are largely ascribed to targeting the imperative macromolecular biosynthetic processes, including cell-wall assembly, DNA replication and transcription, and protein biosynthesis. ${ }^{[9]}$ The misuse and abuse of antibiotics have facilitated bacteria to evolve resistance against almost all the existing antibiotics. ${ }^{[10]}$ The majority of antibiotics routinely used in clinics such as $\beta$-lactam antibiotics, tetracyclines and aminoglycosides, are natural products and their derivatives. Unlike such natural compounds, synthetic chemicals have the potential to circumvent the coevolved resistance to target bacteria with distinctive modes of action. Inspired by the paradigms of sulfonamide and quinolone antibiotics, there are great prospects to mine the unexplored source of abundant synthetic chemicals for antibacterial purposes.

Although synthetic chemicals carrying unique scaffolds always endow them with the potential against MDR bacteria, it suffers from the lack of efficient methodologies to fish the leads, due to the low throughput of common screening assays, e.g. Waksman's platform. Multifunctional chemicals with antibacterial capabilities are potent candidates to advance antibiotic discovery. Recently, aggregation induced emission luminogens (AIEgens), which are weak fluorescent or non-fluorescent in solution but displaying strong fluorescence in aggregated states, ${ }^{[11]}$ have made great progresses in biosensing, ${ }^{[12-13]}$ imaging and antibacterial purposes. ${ }^{[14]}$ Membrane targeting antibacterial agents always increase bacterial metabolic burden with high fitness cost for the development of resistance. ${ }^{[15]}$ Therefore, we hypothesized that the versatile AIEgens are promising leading compounds for screening membrane-targeting antimicrobial agents.

Our previous reports have shown that different AIEgen families are phenomenally active against bacteria, whereas the underlying mechanisms are still unclear. In the present study, we chose TBP molecules (TBPs) as a model to explore their abilities to kill both extracellular and intracellular MDR bacteria. TBPs showed high efficacy against diverse Gram-positive bacteria through membrane disruption and eliminated invased pathogens via enhancing 
autophagy in vitro (Scheme 1) and in an animal model. These findings shed light on the development of next generation of AIEgens to combat MDR bacterial pathogens-assiociated infections.

\section{Results}

\subsection{TBPs effectively kill multidrug-resistant bacteria}

Reports indicated that quaternary ammonium (QA) compounds can effectively kill bacteria and are not easily able to develop resistance. ${ }^{[16]}$ TBP-2 (Figure 1A) exhibited antibiotic activites against both antibiotics-susceptible and MDR Gram-positive bacteria including diverse MRSA and VRE, with the minimal inhibition concentrations (MICs) ranging from $0.25 \mu \mathrm{g} \mathrm{mL}^{-1}$ to 2 $\mu \mathrm{g} \mathrm{mL}^{-1}$ in darkness (Table S1, Supporting Information). However, we found much lower MICs of TBP-1 (Figure 1A) against the same bacteria (Table S1, Supporting Information) with the MICs ranging from $0.0625 \mu \mathrm{g} \mathrm{mL} L^{-1}$ to $0.5 \mu \mathrm{g} \mathrm{mL} L^{-1}$ in darkness (Table S1, Supporting Information). Interestingly, the MICs of such chemcicals decreased 2- to 4-fold under 4 $\mathrm{mW} / \mathrm{cm}^{2}$ light irradiation (Table S1, Supporting Information). Given the unmet and urgent need for treating MRSA-associated infections in clinic, ${ }^{[17]}$ we chose either S. aureus or MRSA for the following mechanistic studies. Bactericidal activities of TBP-2 and TBP-1 were tested by the minimal bactericidal concentration (MBC) assay. ${ }^{[18]}$ The values of $\mathrm{MBC}$ were the same or only 2 fold higher than their corresponding MICs (Figure 1B), which suggests that such chemicals have bactericidal activity. As expected, TBP-1 (10 MIC, $2.5 \mu \mathrm{g} / \mathrm{mL}$ ) killed almost all S. aureus within $1 \mathrm{~h}$ in darkness based on time-kill kinetic analysis (Figure 1C) while TBP2 (10 MIC, $5.0 \mu \mathrm{g} / \mathrm{mL}$ ) killed almost all S. aureus within $3 \mathrm{~h}$ (Figure S1, Supporting Information). Thus, the TBPs can effectively kill antibiotics-susceptible and MDR Grampositive bacteria, TBP-1 have shorter time and lower MIC and MBC than TBP-2. 
A

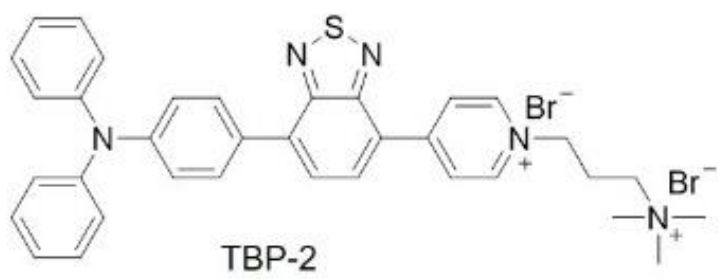

TBP-2

B MBC of AlEgen $(\mu \mathrm{g} / \mathrm{mL})$.

\begin{tabular}{|c|c|c|c|c|c|c|}
\hline \multirow{2}{*}{ AIEgen } & \multicolumn{2}{|c|}{$\begin{array}{c}\text { S. aureus } \\
\text { ATCC } 29213\end{array}$} & \multicolumn{2}{|c|}{$\begin{array}{l}\text { MRSA } \\
\text { QDEF2 }\end{array}$} & \multicolumn{2}{|c|}{ MRSA T144 } \\
\hline & Dark & Light & Dark & Light & Dark & Light \\
\hline TBP-2 & 1 & 0.25 & 2 & 1 & 2 & 1 \\
\hline TBP-1 & 0.25 & 0.0625 & 1 & 0.5 & 0.5 & 0.5 \\
\hline
\end{tabular}
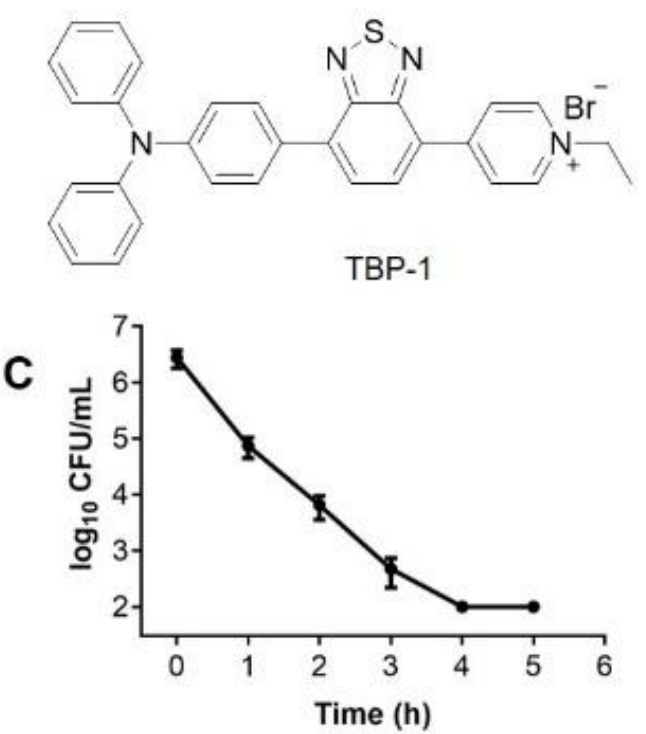

Figure 1. Chemical structure and bactericidal properties of TBP-2 and TBP-1. A) Chemical structure of TBP-2 and TBP-1. B) Bactericidal activities of TBP-2 and TBP-1 with and without light irradiation $(n=3)$, bacterial growth in MHB medium. MBC is the minimal concentration at which less than five colonies grow after the subculture on the agar plate post MICs tests. C) Bactericidal activity of $S$. aureus treated with TBP-1 (10 MICs, $2.5 \mu \mathrm{g} / \mathrm{mL}$ ) as a function of time in darkness $(n=3)$.

\subsection{TBPs trigger membrane damage in Gram-positive bacteria}

Aiming to understand the role of TBPs in killing bacteria. Being consistent with the fact that many antibiotics kill bacteria depending on the generation of ROS, we observed the accumulation of ROS in a dose dependent manner unpon the addtion of TBP-1 (Figure 2A). Increased levels of ROS can cause membrane damage, confirmed by the dissipation of membrane potential in S. aureus treated with TBP-1 (Figure 2B). However, TBP-2 showed low accumulation of intracellular ROS (Figure S2, Supporting Information). To get better understanding of AIEgen-mediated antibacterial activity, we observed that bacteria could be immediately stained by TBPs (Figure S3, Supporting Information). Subsequently, we used TBP-1 as a model to co-stain with commerical membrane (FM $\left.{ }^{\mathrm{TM}} 4-64 \mathrm{FX}\right)$ and well (WGA Alexa Fluor 640) staining dye. Results showed that TBP-1 mainly bound to the cell membrane

(Figure 2C) but not cell wall (Figure S4, Supporting Information) in S. aureus. Co-stainning 
with the commerical dead cell staining dye propidium iodide (PI) results also showed that TBPs disrupted the membrane of S. aureus after incubation for 10 min (Figure S5, Supporting Information). Moreover, we employed the field emission scanning electron microscopy (FESEM) to visualize the morphological changes of bacteria. S. aureus exhibited wrinkling, collasped and lysed structures after treated with TBPs in the darkness, while light irradiation aggravated the morphological changes of the bacteria (Figure 2D). Charge affects the efficiency of molecular entering through cell membrane, TBP-2 needs to overcome larger energy barrier to go through cell membrane and have lower permeability coefficient than TBP-1 (Figure S6, Supporting Information). ${ }^{[19]}$ On the other hand, the hydrophobicity, which can be reflected by the calculated $\log \mathrm{P}$ (n-octanol/water partition coefficient, $\mathrm{C} \log \mathrm{P}$ ) value, can also influence the behavior of the TBP molecules. The ClogP of TBP-2 and TBP-1 was -0.7 and 3.5, repectively. The fact that TBP-2 had lower ClogP than TBP-1 revealed that TBP-2 have better water solubility and need high concentration in aggregate state to kill bacteria. ${ }^{[20]}$ Taken together, these findings indicate that TBPs can target bacteria membrane and the destroy may induce ROS generation to lead to the death of bacteria. In addition, light irradiation can further enhance the accumualtion of ROS in Gram-positive bacteria in the presence of AIEgen, leading to aggravated membrane damage. 

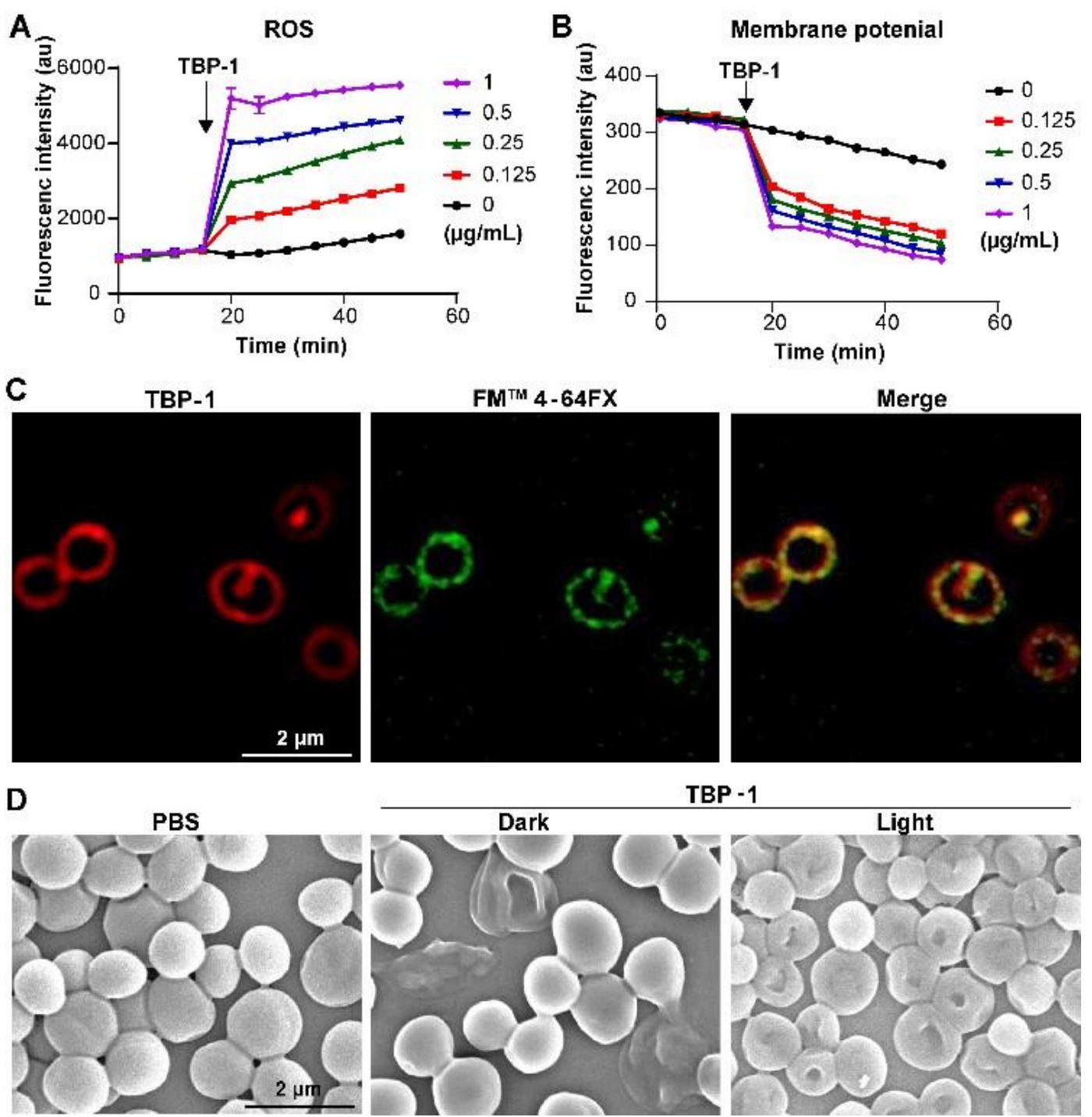

Figure 2. TBP-1 killed $S$. aureus by targeting $S$. aureus membrane. A) Plot of the intracellular ROS fluorescence intensity of $S$. aureus after incubation with TBP-1 $(0.125,0.25,0.5$ and 1 $\mu \mathrm{g} / \mathrm{mL})$ as a function of time. B) Plot of membrane potential $(\Delta \Psi m)$ fluorescence intensity of $S$. aureus after incubation with TBP-1 $(0,0.125,0.25,0.5$ and $1 \mu \mathrm{g} / \mathrm{mL})$ with the time increased. C) Super-resolution SIM images of $S$. aureus incubation with TBP-1 $\left(1 \mu \mathrm{g} / \mathrm{mL}\right.$, red) and $\mathrm{FM}^{\mathrm{TM}}$ 4-64FX ( $5 \mu \mathrm{g} / \mathrm{mL}$, green) for $5 \mathrm{~min}$. TBP-1: $\mathrm{Ex}=488 \mathrm{~nm}, \mathrm{Em}=600-700 \mathrm{~nm}$; FM ${ }^{\mathrm{TM}}$ 4-64FX: $\mathrm{Ex}=640 \mathrm{~nm}, \mathrm{Em}=650-700 \mathrm{~nm} . \mathrm{D})$ Morphology of $S$. aureus incubation with TBP-1 (dark, $0.25 \mu \mathrm{g} / \mathrm{mL}$; light, $0.0625 \mu \mathrm{g} / \mathrm{mL})$ with and without white light irradiation $\left(4 \mathrm{~mW} \mathrm{~cm}^{-2}\right)$.

\subsection{TBPs kill intracellular $S$. aureus through enhancing autophagy}

In view of the promising bactericidal activity of TBPs, we tested their antibacterial ability against intracellular bacteria. Compared to TBP-2, TBP-1 exhibited lower values of MIC and MBC against MRSA isolates under light irradiation (Table S2, Table S3, Supporting 
Information). Also, TBP-1 has low toxicity with high therapeutic index (Table S4, Supporting Information). As expected, the selective killing of bacteria was demonstrated and the mammalian cell viability was essentially unaffected. To test whether TBP-1 can target intracellular bacteria, we infected IEC-6 cells with $S$. aureus at multiplicity of infection (MOI) of 1. Infection rate of intracellular bacteria increased in a time dependent manner (Figure S7A, Figure S7B, Supporting Information). The number of intracellular bacteria decreased sharply under the treatment of TBP-1, which was further enhanced under light irradiation (Figure 3A). Autophagy, an evolutionarily conserved process, plays an essential role in enabling eukaryotic organisms to clear bacterial invasion. ${ }^{[21]}$ To determine whether autophagy contributes to TBPsmediated killing of intracellular bacteria, we evaluated the expression levels of autophagyrelated proteins, including LC3-II, LC3-I, and p62 in IEC-6 cells infected with S. aureus. ${ }^{[22]}$ We found increased ratio of LC3-II/LC3-I and reduced level of p62 protein, with an increase of infection time (Figure S7C, Supporting Information). Activated autophagy was further supported by the test with chloroquine (CQ), a lysosome inhibitor to specifically block the autophagosome-lysosome fusion step (Figure S7D, Supporting Information). These findings indicate that autophagy is induced in IEC-6 cells infected with $S$. aureus, being consistent with previous reports. ${ }^{[23]}$ It has been reported that some bacteria can persist in the cytoplasm of mammalian cells by hijacking autophagy. ${ }^{[24]}$ We observed that the number of intracellular $S$. aureus increased with the co-culture of $\mathrm{CQ}$, while autophagy activator rapamycin (Rapa) reduced the number of intracellular bacteria (Figure S7E, Supporting Information). In addition, S. aureus escaped from the degraded lysosomes, indicating the inhibition of lysosomal acidification (Figure S7F, Supporting Information). Together, these results suggest that $S$. aureus survival and replicate in IEC-6 cells through hijacking autophagy. To gain detailed understanding of TBP-1 killing intracellular S. aureus, we dissected the effect of TBP-1 on the process of autophagy in IEC-6 cells infected with S. aureus. TBP-1 treatment boosted autophagy by increasing the ratio of LC3-II/LC3-I and reducing the level of p62 protein (Figure 
3B, Figure S8, Supporting Information). Compared to the treatment of CQ, we observed the decrease of the number of intracellular bacteria treated with TBP-1 (Figure 3C). Furthermore, we found that the co-localization of TBP-1 with lysosome and S. aureus of IEC-6 cells (Figure 3D). It suggested that TBP-1 killed intracellular S. aureus mainly in lysosome. The western blot results showed that the ratio of LC3-II/LC3-I increased and the level of p62 protein reduced after TBP-2 treatment (Figure S9, Supporting Information). Taken together, these results suggest that TBPs clear and kill intracellular S. aureus through enhanced autophagy of mammalian cells.
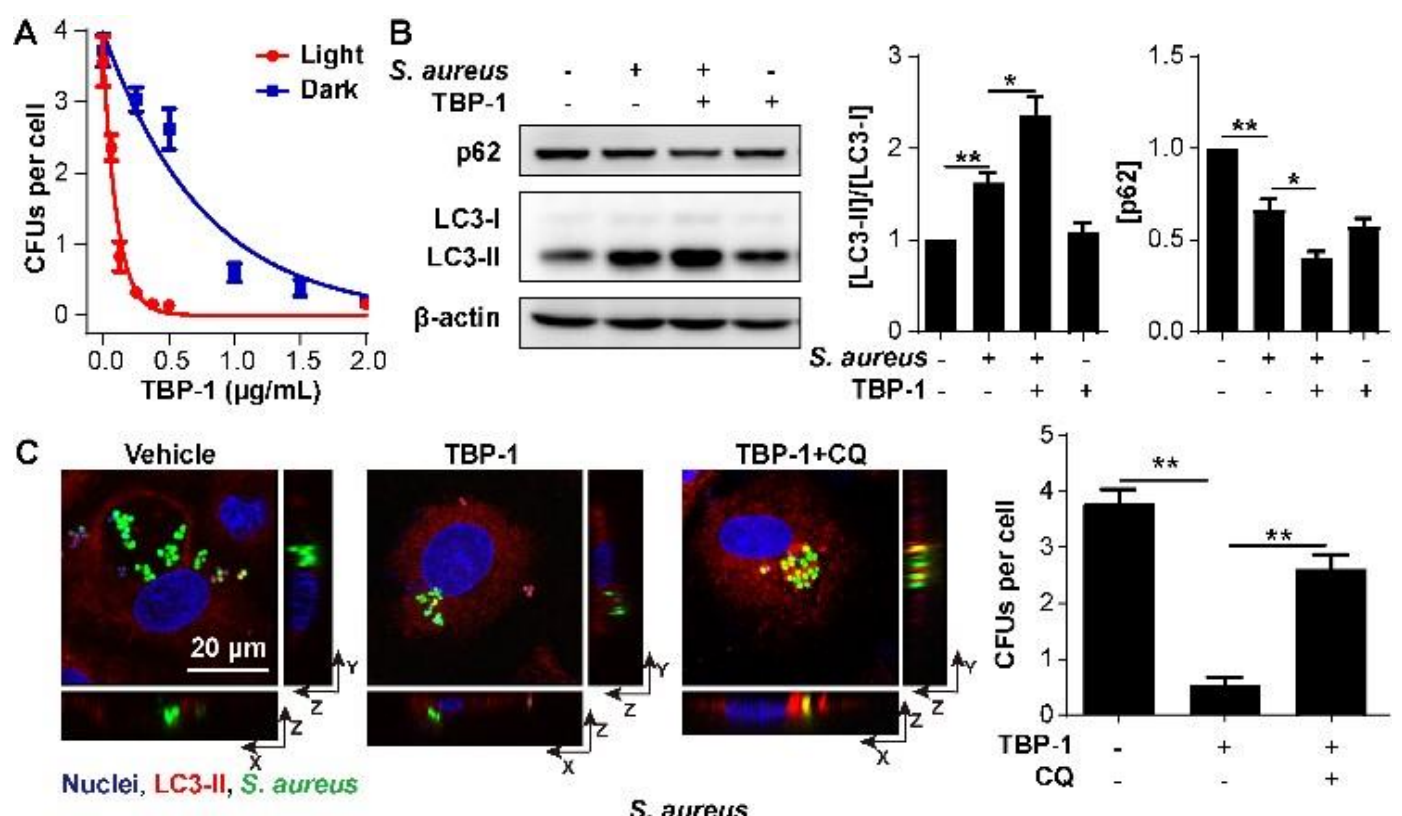

D

TBP-1
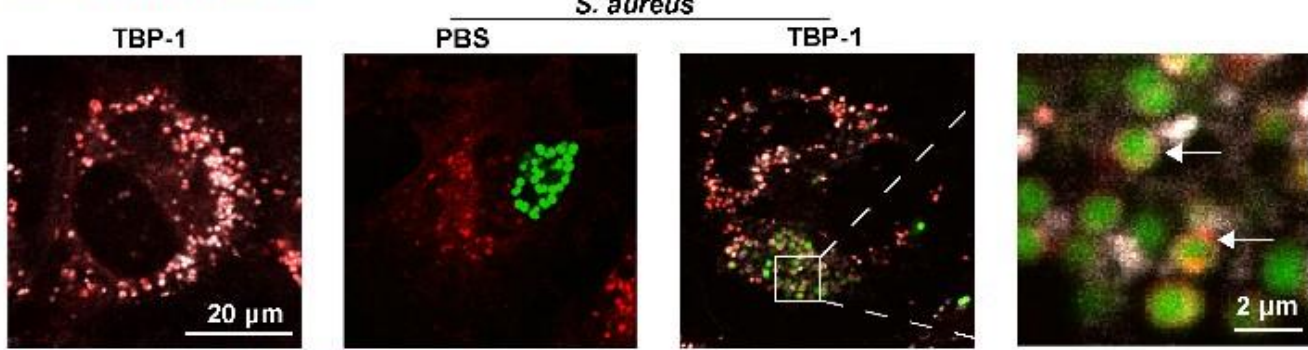

S. aureus, TBP-1, Lysotracker red

Figure 3. TBP-1 killed intracellular $S$. aureus through enhancing autophagy of IEC-6 cells. A) Bactericidal activity of intracellular $S$. aureus treated with different concentrations of TBP-1 with and without light irradiation. B) Western blot analysis of Microtubule Light Chain 3 (LC3) and p62 expression in IEC-6 cells infected with or without $S$. aureus in the presence and absence of TBP-1 $(1 \mu \mathrm{g} / \mathrm{mL})$. All proteins were normalized to the level of $\beta$-actin. C) Fluorescent images and CFUs of intracellular S. aureus after IEC-6 cells incubated with S. aureus, S. aureus + TBP1 and a lysosome inhibitor chloroquine $(\mathrm{CQ})+S$. aureus + TBP-1. IEC-6 cells were stained 
with LC3 $(E x=552 \mathrm{~nm}, \mathrm{Em}=565 \mathrm{~nm})$ and DAPI $(E x=405 \mathrm{~nm}, \mathrm{Em}=454 \mathrm{~nm})$. D) Fluorescent images of IEC-6 cells treated with TBP-1, S. aureus (green) + PBS, S. aureus + TBP-1, and then stained with lysotracker red $(E x=552 \mathrm{~nm}, \mathrm{Em}=590 \mathrm{~nm})$. Magnification images of the outlined area are shown on the right, the white arrows indicate the overlay of lysotracker, TBP1 and $S$. aureus. $n s$ not significantly, $* P<0.05, * * P<0.001$.

\subsection{ROS play a key role in TBPs-induced killing of intracellular S. aureus}

To further understand how TBP-1 enhanced autophagy, we hypothesized that mitochondrial perturbation contributes to the clearance of the invaded bacteria. We found that S. aureus slightly caused morphological damage to mitochondria, however, the addition of TBP-1 targeted mitochondria and dramatically aggravated such damage (Figure 4A). Damaged mitochondria resulted in the accumulation of intracellular ROS (Figure 4B). Similarly, TBP-2 incubation can increase the intracellular ROS levels (Figure S10, Supporting Information). To clarify the relative mechanisms, we further investigated the mitochondrial membrane potential $(\Delta \Psi \mathrm{m})$ in IEC-6 cells based on the cyanine dye $5,5^{\circ}, 6,6^{\prime}$-tetrachloro-1,1',3,3' tetraethylbenzimidazolyl- carbocyanine iodide (JC-1) staining assay. ${ }^{[25]}$ We observed the increase of the formation of green fluorescent JC-1 monomers (Figure S11, Supporting Information) and increased ratio of green/red fluorescence under the treatment of TBP-1 (Figure S11B, Supporting Information). These results suggest that TBP-1 paralyzed the homeostasis of mitochondria, which may increase the production of intracellular ROS. Exogenous addition of ROS scavenger $N$-acetyl cysteine (NAC) significantly blocked the

clearance of intracellular S. aureus under TBP-1 treatment (Figure S12, Supporting Information). It indicates that ROS plays a critical role in TBP-1-mediated clearance of invaded S. aureus. Furthermore, we quantified the amount of TBP-1 in the cytosol of IEC-6 cells infected with $S$. aureus based on liquid chromatography-tandem mass (LC-MS/MS) analysis (Figure S13, Table S5, Supporting Information). The accumulated intracellular TBP-1 
consisted about $6 \%$ of the total TBP-1 (Figure 4C), which can be easily enriched to high levels in the limited space in mammalian cells to clear bacteria.

A
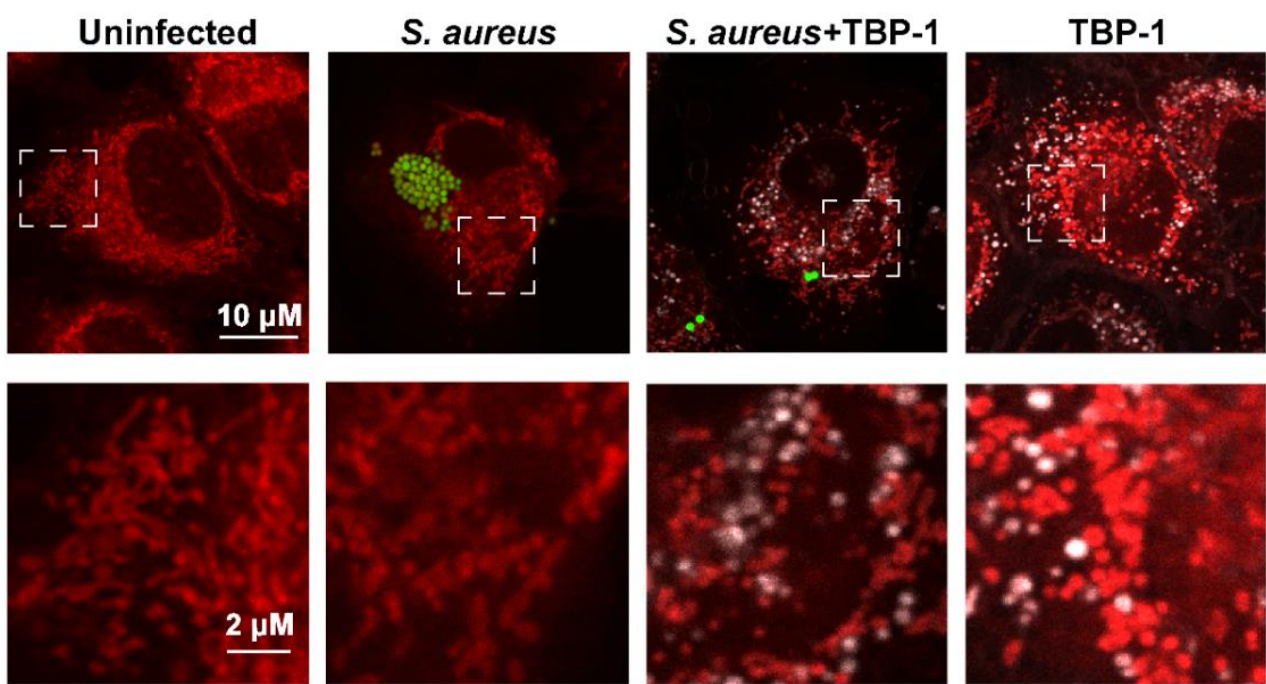

S. aureus, TBP-1, Mitotracker red

B

C

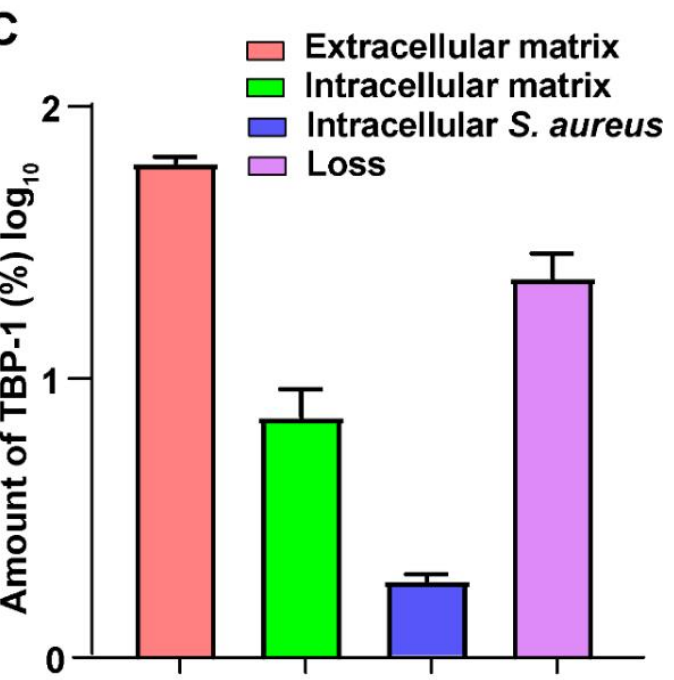

S. aureus TBP-1 -

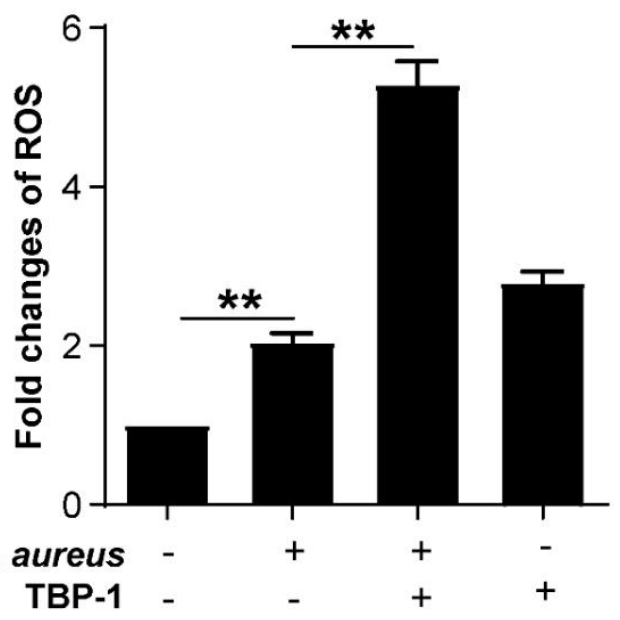

Figure 4. TBP-1 enhanced autophagy through inducing mitochondrial dysfunction. A) Morphology and structure changes of mitochondria after IEC-6 cells incubated with and without S. aureus (green), S. aureus + TBP-1 and TBP-1. The mitochondria stained by Mitotracker red $(\mathrm{Ex}=552 \mathrm{~nm}, \mathrm{Em}=600 \mathrm{~nm})$. The white rectangles indicate the magnified part of the bottom images. B) Fold changes of ROS in IEC-6 cells infected with or without $S$. aureus in the presence and absence of TBP-1 $(* * P<0.001)$. C) Relative amount of TBP-1 in medium, IEC-6 cells, $S$. aureus and lost in in the process of experimental treatment. The quantified of TBP-1 was measured by LC-MS/MS.

\subsection{TBPs protect mice from MRSA associated infection}


Given the promising antibacterial activity of TBPs in vitro, we evaluated its therapeutic effect on mouse peritonitis model. Mice were injected intraperitoneally with MRSA T144 with single dose that can lead to $90 \%$ lethality. TBPs were introduced intraperitoneally at one-hour postinfection. The infected mice without treatment all died within $48 \mathrm{~h}$, while the mice survived under the treatment of either TBP-1 (Figure 5A) or vancomycin $(5 \mathrm{mg} / \mathrm{kg}$ ) (Figure 5B). Meanwhile, bacterial counts in different organs decreased significantly in the presence of 2.5 mg/kg TBP-1 (Figure 5A), which showed comparable survival rates of bacteria in different organs treated with vancomycin $(10 \mathrm{mg} / \mathrm{kg})$ (Figure 5B). Similarly, all mice survived in the presence of $5 \mathrm{mg} / \mathrm{kg}$ TBP-2 (Figure S14A, Supporting Information), with decreased bacterial counts in organs (Figure S14B, Supporting Information). Lastly, typical pathologic changes of the organs were alleviated in the treated groups as well (Figure 5C). However, the organs showed hemorrhage and congestion after T144 infection, especially the spleen and lung (Figure 5C, Figure S14C, Supporting Information). Together, these results demonstrate that AIEgens such as TBP-1 are potent agents for the treatment of MRSA associated infections in vivo.

\section{Discussion}

Antibiotics that target bacterial membrane always increase metabolic burden, therefore it is not easy to develop antibiotic resistance due to high fitness cost. ${ }^{[26]}$ However, membrane-targeting antibiotics have been rarely exploited. Similar to synthetic antibacterial compounds derived from sulfonamides and quinolones, ${ }^{[27]}$ the versatile skeletons of AIEgens will provide tremendous sources for developing lead compounds to circumvent antibiotic resistance with distinctive mechanisms. Notably, the intrinsic fluorescent property of AIEgens offers great potentials to track and dissect their modes of action in bacteria and pharmacokinetics processes in hosts. We get insightful understandings of TBPs-mediated antibacterial activities, which directly disrupts bacterial membrane and indirectly potentiates host cells to clean invasive bacteria. 
A
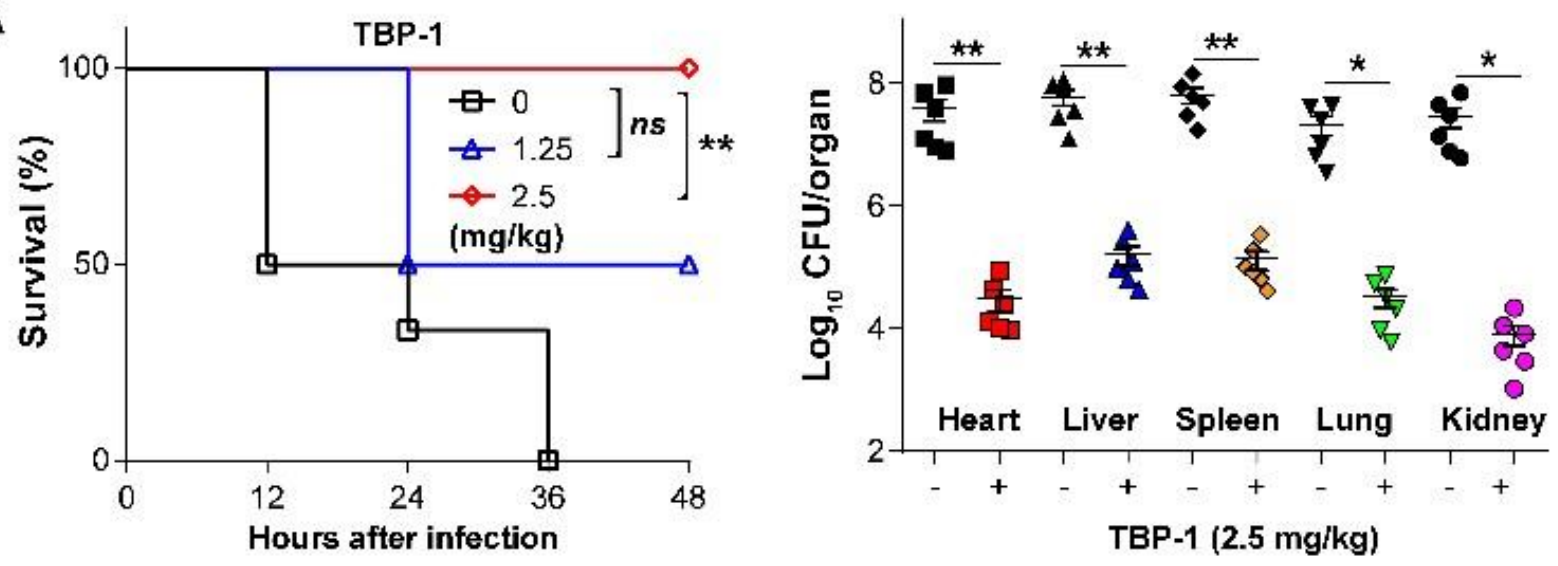

B
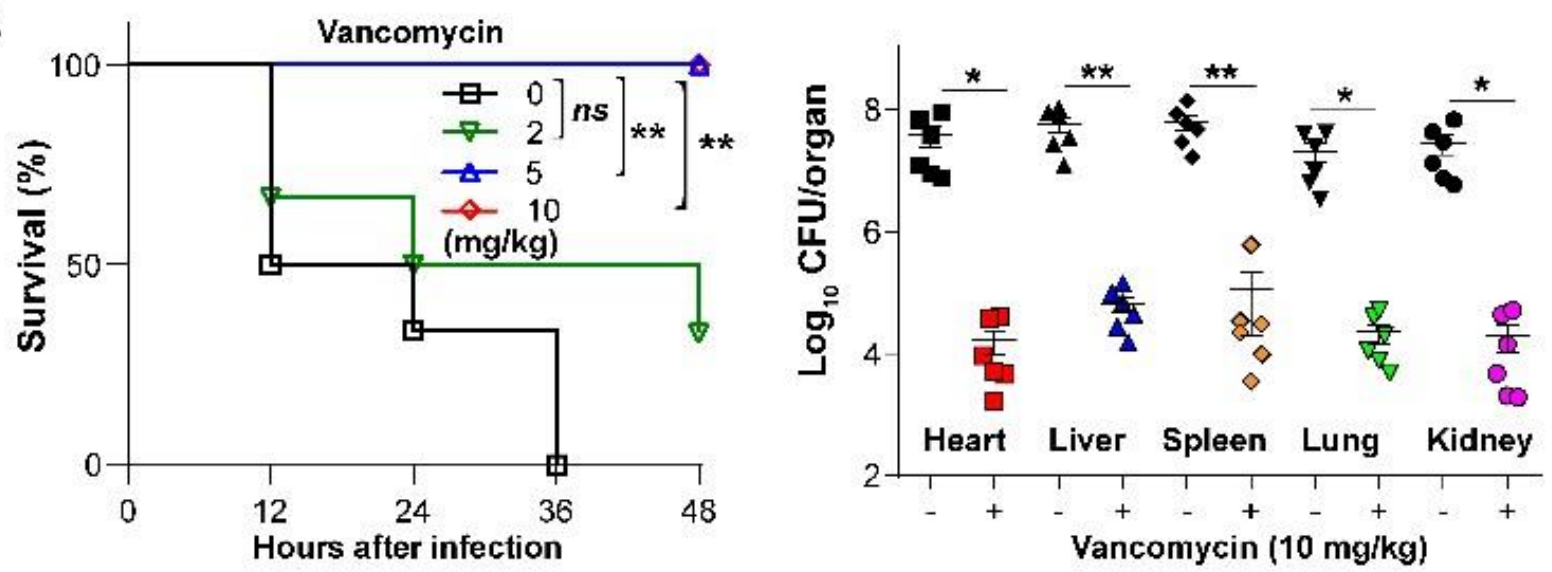

C Heart Liver
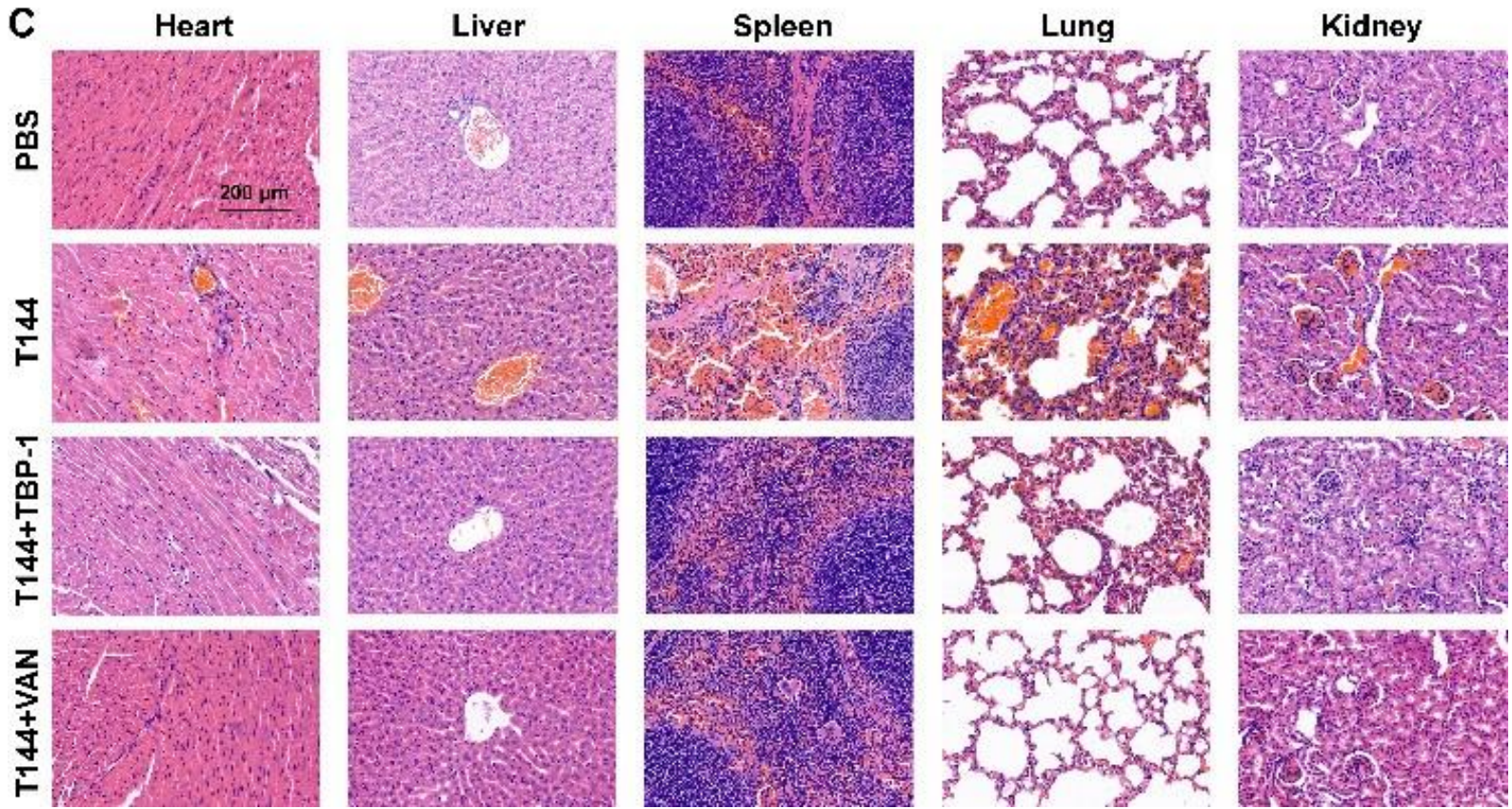

Figure 5. Efficacy of TBP-1 and vancomycin in the mouse peritonitis model. Survival rate and bacteria survival in different organs of mice after treatment with TBP-1 (A) and Vancomycin (B) in septicemia protection model using MRSA T144 ( $\mathrm{n}=5, n s$ not significantly; $* P<0.05$; $* * P<0.001)$. C) Histological hemotoxylin and eosin (HE) staining images of Heart, liver, spleen, lung and kidney tissue sections at day 2 after different treatments indicated. 
The cell wall of Gram-positive bacteria is composed of cross-linked peptidoglycans and anionic teichoic acids. Cationic TBPs may bind to the negatively charged teichoic acids, facilitating the insertion into the porous structure of cell wall to further disrupt membrane integrity to cause bacterial death. Nevertheless, such electrostatic interaction can be impaired in Gram-negative bacteria, due to the presence of thick and highly hydrophobic layer of lipopolysaccharide. Compared to TBP-1, consistently, high concentrations of TBP-2, with more net positive charges, was effective against E. coli (Table S6, Supporting Information). This observation agrees with our previous findings that adequate modification of certain groups (hydrophilic amines, ${ }^{[28]}$ naphthalimide triazole, ${ }^{[14]}$ antimicrobial peptide ${ }^{[29]}$ ) in the AIEgen skeletons promote antibacterial activities against Gram-negative pathogens. Altogether, it suggests that the pyridine ring of TBP is a potential position to introduce functional groups to extend the antibacterial spectrum.

Unlike antibiotics that directly target pathogens, host-directed antibacterial therapy is an alternative approach to potentiate hosts for further elimination of invading bacteria. ${ }^{[30]}$ Notably, autophagy, as a ubiquitous response in host cells, plays a pivotal role in the defense against the invasion of bacterial pathogens. ${ }^{[31]}$ In this study, TBPs not only trigger ROS mediated membrane damage to kill bacteria, but also activate mitochondria-dependent autophagy process to eliminate intracellular bacteria in host cells. In this scenario, versatile TBPs serve as a novel class of antibiotic adjuvants to boost mammalian cells for bacterial clearance. Future studies are urgently required to demonstrate that whether and how such capability of antibiotic adjuvant works in vivo under pathophysiological conditions, guiding the optimization of the structureactivity relationship of AIEgen molecules for tracing and killing MDR bacterial pathogens

\section{Experimental Section}

Materials: All bacterial strains used in this study are listed in Table S1(Supporting Information) and Table S6 (Supporting Information). Bacteria were grown in brain heart infusion (BHI) broth (Land Bridge Technology) or on BHI agar plates at $37^{\circ} \mathrm{C}$. IEC-6, A549, 
HUVEC, and 3T3 cells (Table S7, Supporting Information) were grown in Dulbecco's Modified Eagle Medium (DMEM, Gibco) supplemented with $1 \%$ heat inactivated fetal bovine serum (FBS, Invitrogen), 1\% (w/v) penicillin-streptomycin and 1\% (w/v) sodium pyruvate (SigmaAldrich). Vancomycin were obtained from China Institute of Veterinary Drug Control.

Antimicrobial Assay: MICs of TBPs were determined by broth micro-dilution according to the Clinical and Laboratory Standards Institute (CLSI) 2015 guideline. ${ }^{[32]}$ After $16-20 \mathrm{~h}$ incubation at $37^{\circ} \mathrm{C}$, MICs values were defined as the lowest concentrations of antibiotics with no visible growth of bacteria. The bacteria colonies grown on the agar plate were counted and MBC (less than five colonies) of each sample was obtained after MICs tests. ${ }^{[33]}$

Time-dependent killing kinetic profiles were performed on the basis of a previous study. ${ }^{[18]}$ Overnight culture of S. aureus ATCC 29213 was 10,000 folds diluted in $1 \mathrm{~mL}$ MHB medium and incubated at $37^{\circ} \mathrm{C}$ with shaking at $200 \mathrm{rpm}$. Bacterial populations at exponential phase (4 h) were challenged with TBPs at concentrations corresponding to the 10 - fold MICs $(2.5 \mu \mathrm{g} / \mathrm{mL}$ TBP-1, $5 \mu \mathrm{g} / \mathrm{mL}$ TBP-2). After incubation at $37{ }^{\circ} \mathrm{C}$ with shaking at $200 \mathrm{rpm}$, serially diluted suspensions at different time points were plated on S. aureus chromogenic agar plates (CHROM agar) and incubated at $37{ }^{\circ} \mathrm{C}$ for overnight. Colonies were counted and colony forming unit $\left(\mathrm{CFU} \mathrm{mL} \mathrm{mL}^{-1}\right)$ were calculated. Experiments were performed with three replicates.

ROS detection: Intracellular ROS were detected by ROS Assay Kit (Beyotime, China). The 2,7Dichlorodi-hydrofluorescein diacetate (DCFH-DA) turn to 2,7-Dichlorofluorescein (DCF) (green fluorescence) represented ROS release $(\mathrm{Ex}=488 \mathrm{~nm}, \mathrm{Em}=525 \mathrm{~nm})$. Intracellular ROS fluorescence intensity of $S$. aureus after incubation with TBP-1 (0, 0.125, 0.25, 0.5 and 1 $\mu \mathrm{g} / \mathrm{mL})$, or TBP-2 $(0,0.25,0.5,1$ and $2 \mu \mathrm{g} / \mathrm{mL})$ from 0 to 180 min were measured on a microplate reader (Tecan, Infinite 200 Pro Microplate Reader, Switzerland). Intracellular ROS fluorescence intensity in IEC-6 cells infected with or without $S$. aureus in the presence and absence of TBP-1 $(1 \mu \mathrm{g} / \mathrm{mL})$ or TBP-2 $(2 \mu \mathrm{g} / \mathrm{mL})$ were also measured on a microplate reader. 
Bacteria membrane potential Assay: Membrane permeability and dissipated membrane potential ( $\Delta \Psi m)$ of $S$. aureus ATCC 29213 induced by TBP-1 $(0.125,0.25,0.5,1 \mu \mathrm{g} / \mathrm{mL})$ were tested by the fluorescent dyes (10 nmol/L) 3, 3-dipropylthiadicarbocyanine iodide ( $\left.\operatorname{DiSC}_{3}(5)\right)$ (Aladdin) according to a previous report. ${ }^{[34]}$ Experiments were performed with three replicates. Membrane permeability test: Membrane permeability of S. aureus ATCC 29213 induced by TBPs were tested by the fluorescent dye $(10 \mathrm{nmol} / \mathrm{L}$ ) propidium iodide (PI) (Aladdin) according to a previous report. ${ }^{[35]}$

Cell viability assay: IEC-6, A549, HUVEC, and 3T3 cells were cultured in DMEM medium, which contained $1 \%$ FBS, $1 \%(\mathrm{w} / \mathrm{v})$ penicillin-streptomycin and $1 \%(\mathrm{w} / \mathrm{v})$ sodium pyruvate. Cells were seeded in 96-well plates $\left(0.5 \times 10^{4}\right.$ cells $\left.200 \mu \mathrm{L}^{-1}\right)$ and incubated for $24 \mathrm{~h}$. The medium was removed and TBPs was added at different concentrations and incubated in darkness for $30 \mathrm{~min}$ at $37^{\circ} \mathrm{C}$. The TBPs-treated cells were exposed to white light for $30 \mathrm{~min}$, and the TBPs-treated cells in darkness were set as control. The cell medium was replaced by fresh medium and incubated for $24 \mathrm{~h}$ at $37{ }^{\circ} \mathrm{C}$ after irradiation. $100 \mu \mathrm{L}$ of fresh medium containing $10 \mu \mathrm{L}$ WST-1 (Roche, Germany) solution was added into each well after the removal of the cell medium, and the cells were incubated at $37^{\circ} \mathrm{C}$ for $1 \mathrm{~h}$. The absorption of the samples was measured on a microplate reader (Tecan, infinite M200, Switzerland) at $450 \mathrm{~nm}$. Cell viability $\left(\mathrm{IC}_{50}\right)$ was determined by the concentrations of analytes of target resulting in $50 \%$ inhibition of cell growth.

Bacteria culturing, staining, and imaging: S. aureus ATCC 29213 were cultured in the BHI broth medium at $37^{\circ} \mathrm{C}$ with a shaking speed of $200 \mathrm{rpm}$ for $3 \mathrm{~h}$. Bacteria were harvested by centrifuging at $8000 \mathrm{rpm}$ for $3 \mathrm{~min}$ and washed twice with PBS. $1 \mathrm{~mL}$ of TBP-1 $(1 \mu \mathrm{g} / \mathrm{mL})$ or TBP-2 $(1 \mu \mathrm{g} / \mathrm{mL})$ solution in BHI broth medium containing $5 \times 10^{8}$ colony forming unit $(\mathrm{CFU}$ $\mathrm{mL}^{-1}$ ) of bacteria incubated at $37{ }^{\circ} \mathrm{C}$ with a shaking speed of $200 \mathrm{rpm}$ for $5 \mathrm{~min}$. To take fluorescence images, $2 \mu \mathrm{L}$ of stained bacteria solution was transferred to a piece of glass slide and then covered by a coverslip. The images were collected using a Stimulated Emission of 
Depletion (STED) microscopy (Leica SP8 STED 3X, Germany). TBP-1 (1 $\mu \mathrm{g} / \mathrm{mL})$ and FM464FX $(5 \mu \mathrm{g} / \mathrm{mL})$ containing $5 \times 10^{8} \mathrm{CFU} \mathrm{mL} \mathrm{m}^{-1}$ of bacteria incubated at $37{ }^{\circ} \mathrm{C}$ with a shaking speed of $200 \mathrm{rpm}$ for $5 \mathrm{~min} . \mathrm{TBP}-1(1 \mu \mathrm{g} / \mathrm{mL})$ and WGA Alexa Fluor $640(5 \mu \mathrm{g} / \mathrm{mL})$ containing $5 \times 10^{8} \mathrm{CFU} \mathrm{mL} \mathrm{m}^{-1}$ of bacteria incubated at $37^{\circ} \mathrm{C}$ with a shaking speed of $200 \mathrm{rpm}$ for $10 \mathrm{~min}$. To take fluorescence images, $2 \mu \mathrm{L}$ of stained bacteria solution was transferred to a piece of glass slide and then covered by a coverslip. The images were collected using Super-Resolution SIM Microscopy (N-SIM/N-STORM010899, Nikon, Japan).

FE-SEM analysis: Bacterial cultures of S. aureus ATCC 29213 at mid-log phase in BHI medium were collected and re-suspended into PBS at cell density of approx. $10^{10} \mathrm{CFU} \mathrm{mL} \mathrm{m}^{-1}$. After treatment of bacteria with TBP-1 (dark, $0.25 \mu \mathrm{g} / \mathrm{mL}$; light, $0.0625 \mu \mathrm{g} / \mathrm{mL}$ ) and TBP-2 (dark, $1 \mu \mathrm{g} / \mathrm{mL}$; light, $0.25 \mu \mathrm{g} / \mathrm{mL}$ ) with or without light irradiation for $30 \mathrm{~min}$, bacteria were collected and fixed in $2.5 \%$ glutaraldehyde solution. After fixation, samples were washed with $0.1 \mathrm{M}$ phosphate buffered saline (PBS) and dehydrated by 30\%, 50\%, 70\%, 80\%, 90\%, 95\%, and $100 \%$ (v/v, in water) ethanol for analysis by field emission scanning electron microscopy (FE-SEM) (S4800, Hitachi, Japan).

Mechanism of killing intracellular bacteria analysis: IEC-6 cells were pro-treated with chloroquine (CQ) for $3 \mathrm{~h}$, then IEC-6 cells were infected with $S$. aureus at multiplicity of infection (MOI) of 1 for $4 \mathrm{~h}, S$. aureus were removed, then cells were treated with $1 \mu \mathrm{g} / \mathrm{mL}$ TBP-1 for $2 \mathrm{~h}$, last cells were then stained with LC3 $(\mathrm{Ex}=552 \mathrm{~nm}, \mathrm{Em}=565 \mathrm{~nm})$ and DAPI $(\mathrm{Ex}=405 \mathrm{~nm}, \mathrm{Em}=454 \mathrm{~nm})$, the number of intracellular $S$. aureus was counted by plate. IEC6 cells were pro-treated with $N$-acetyl cysteine (NAC) for $3 \mathrm{~h}$, then IEC- 6 cells were infected with $S$. aureus at MOI of 1 for 4 h, S. aureus were removed, then cells were treated with 1 $\mu \mathrm{g} / \mathrm{mL}$ TBP-1 for $2 \mathrm{~h}$, last cells were then stained with DAPI $(\mathrm{Ex}=405 \mathrm{~nm}, \mathrm{Em}=454 \mathrm{~nm})$ and F-actin $(E x=552 \mathrm{~nm}, \mathrm{Em}=565 \mathrm{~nm})$, the number of intracellular $S$. aureus was counted by plate. For static images, fixed and stained intestinal or cellular samples were captured by a Leica SP8 confocal microscope (Leica, Gremany). 3D images were taken by capture all the X-, Y- 
and Z-axis sections. For analyzing the location of intracellular bacteria, the Z-axis section was cut every $1 \mu \mathrm{m}$ or $2 \mu \mathrm{m}$. Images were analyzed and merged by the LAS AF Lite software (Leica, Gremany). Cells were treated as same, and then were tracked by lysotracker red, and cells were observed through confocal microscope. IEC-6 cells were infected with $S$. aureus at MOI of 1 for $4 \mathrm{~h}, S$. aureus were removed, then cells were treated with $1 \mu \mathrm{g} / \mathrm{mL}$ TBP-1 for $2 \mathrm{~h}$. The mitochondria of IEC-6 were tracked by Mito-tracker $(\mathrm{Ex}=552 \mathrm{~nm}, \mathrm{Em}=600 \mathrm{~nm})$. The morphological change was observed by confocal microscopy. The ROS of IEC-6 were tracked by ROS assay kit. ROS change was observed by microplate reader (Tecan, infinite M200, Switzerland $)(\mathrm{Ex}=488 \mathrm{~nm}, \mathrm{Em}=525 \mathrm{~nm})$.

Western blot: IEC-6 cells were infected with S. aureus at MOI of 1 for $4 \mathrm{~h}$, S. aureus were removed, then cells were treated with $1 \mu \mathrm{g} / \mathrm{mL}$ TBP-1 $(1 \mu \mathrm{g} / \mathrm{mL}$ TBP- 2$)$ for $2 \mathrm{~h}$, then the level of LC3 and p62 were tested by Western blot. CQ $(50 \mu \mathrm{g} / \mathrm{mL}) /$ Rapa $(100 \mathrm{nM})$ was used to treat IEC-6 cells before $S$. aureus infected. All proteins were normalized to the level of $\beta$-actin. The primary antibodies included rabbit anti-LC3B, anti-p62 (Abcam, UK) and mouse anti- $\beta$-actin antibodies (Proteintech, USA), secondary antibodies were goat anti-rabbit and goat anti-mouse antibodies (Beyotime, China). Gray values of protein bands were quantified by Image J software.

Mitochondrial membrane potential assay: The mitochondrial membrane potential $(\Delta \Psi \mathrm{m})$ in IEC- 6 cells based on the cyanine dye JC- 1 staining assay. JC-1 aggregates in the mitochondrial matrix to form a polymer, which emits a strong red fluorescence. Due to the decrease or loss of membrane potential in unhealthy mitochondria, JC-1 can only exist as a monomer in the cytoplasm, producing green fluorescence. IEC-6 cells were infected with $S$. aureus at MOI of 1 for 4 h treated with or without TBP-1, IEC- 6 cells treated with TBP-1 and IEC- 6 cells were stained with JC-1, respectively. The imaging was captured by a Leica SP8 confocal microscope (Leica, Gremany), and Green and red fluorescence ratio of ICE-6 cells analyzed by Image J. 
Distribution of TBP-1: The concentrations of extracellular TBP-1, intracellular TBP-1 in the cytosol of IEC-6 cells, TBP-1 in S. aureus and loss antibiotics were quantified by liquid chromatography-tandem mass (LC-MS/MS).

Mouse sepsis protection model: 6-8 weeks old female BALB/c mice were purchased from the Vital River Laboratory Animal Technology Co. Ltd., (Beijing, China) and housed under specified pathogen-free conditions for one week. The in vivo bioavailability of TBP-1 and TBP2 was tested against a clinical isolate MRSA T144 in a mouse sepsis protection model according to a previous protocol. ${ }^{3}$ Briefly, seven groups of BALB/c female mice $(n=6)$ were infected intraperitoneally with $0.5 \mathrm{~mL}$ of bacterial suspension $\left(7.5 \times 10^{8} \mathrm{CFU}\right.$ per mouse $)$ and monitored daily for survival. After $1 \mathrm{~h}$ post-infection, mice in each group were treated with TBP-1 at single intraperitoneal doses of 1.25 and $2.5 \mathrm{mg} / \mathrm{kg}$, while TBP-2 at dose of 2.5 and $5 \mathrm{mg} / \mathrm{kg}$. Additionally, two groups were treated with vancomycin at doses of 2,5 and $10 \mathrm{mg} / \mathrm{kg}$ as positive controls. ${ }^{[36]}$ For determination of the bacterial burden, mice were sacrificed at $48 \mathrm{~h}$. Heart, liver, spleen, lung and kidney were collected and homogenized in sterile PBS. Serial dilutions of each suspension were plated on S. aureus chromogenic agar plates (CHROM agar) for the enumeration of bacterial colonies.

Hematoxylin-eosin (HE) staining: Samples of mouse organs were excised and fixed with $4 \%$ paraformaldehyde solution (Sigma). Histological images were taken using an inverted microscope (Olympus, IX71, Japan). Histopathological changes were analyzed by a traditional hematoxylin-eosin (HE) staining protocol.

Ethics statement: Animal experiments were performed in strict accordance with the regulations for the Administration of Affairs Concerning Experimental Animals approved by the State Council of People's Republic of China (11-14-1988). The animal study protocols were performed in accordance with the relevant guidelines and regulations (ID: SKLAB-B-2010003). The laboratory animal usage license number is SYXK-2016-0008, certified by Beijing Association for Science and Technology. 
Statistical analysis:The values reported are expressed as mean standard deviation (SD). The Origin 8 software was used for graph plotting. A value of $P<0.05$ was considered significant. Each experiment included at least three replicates.

\section{Supporting Information}

Supporting Information is available from the Wiley Online Library or from the author.

\section{Acknowledgements}

Y. Li and F. Liu contributed equally to this work. We are grateful for financial support from the Research Grants Council of Hong Kong (16305618, 16305518 and C6009-17G). the Innovation and Technology Commission (ITC-CNERC14SC01), National Key Research and Development Program of China (2017YFC1600305, 2018YFE0190200), the National Key Research and Development Program of China (2017YFC1600305), National Natural Science Foundation of China (31922083, 31772796) and the Fund of Beijing Dairy Industry Innovation Team. Natural Science Foundation for Distinguished Young Scholars of Guangdong Province (2020B1515020011), the Natural Science Foundation of China (21801169), the Science and Technology Foundation of Shenzhen City (JCYJ20190808153415062), and Natural science foundation of Shenzhen University (2019004).

\section{References}

[1] K. Kupferschmidt, Science 2016, 352, 758.

[2] Y. Liu, S. Y. Ding, J. Z. Shen, K. Zhu, Nat. Prod. Rep. 2019, 36, 573.

[3] M. L. Lambert, C. Suetens, A. Savey, M. Palomar, M. Hiesmayr, I. Morales, A. Agodi, U. Frank, K. Mertens, M. Schumacher, M. Wolkewitz, Lancet infect. Dis. 2011,11, 30.

[4] J. Hui, P. T. Dong, L. J. Liang, T. Mandal, J. J. Li, E. R. Ulloa, Y. W. Zhan, S. Jusuf, C. Zong, M. N. Seleem, G. Y. Liu, Q. Cui, J. X. Cheng. Adv. Sci. 2020, 7, 1903117.

[5] M. McKenna, https://www.wired.com/2013/09/cdc-amr-rpt1/ 2013.

[6] J. L. Martínez, F. Baquero, D. I. Andersson, Nat. Rev. Microbiol. 2007, 5, 958.

[7] G. E. Thwaites, V. Gant, Nat. Rev. Microbiol. 2011, 9, 215. 
[8] Z. Q. Yang, Y. L. Huang, H. W. Zhou, R. Zhang, K. Zhu, Lancet Infect. Dis. 2017, 18, 22.

[9] M. A. Kohanski, D. J. Dwyer, B. Hayete, C. A. Lawrence, J. J. Collins, Cell 2007, 130, 797.

[10] C. Walsh, Nat. Rev. Microbiol. 2003, 1, 65.

[11] J. D. Luo, Z. L. Xie, J. W. Y. Lam, L. Cheng, H. Y. Chen, C. F. Qiu, R. T. K. Kwork, X. W. Zhan, Y. Q. Liu, D. B. Zhu, B. Z. Tang, Chem. Commun. 2001, 1740.

[12] C. C. Zhou, W. H. Xu, P. B. Zhang, M. J. Jiang, Y. C. Chen, R. T. K. Kwok, M. M. S. Lee, G. G. Shan, R. L. Qi, X. Zhou, J. W. Y. Lam, S. Wang, B. Z. Tang, Adv. Funct. Mater. 2019, 29, 1805986.

[13] B. Situ, X. Y. Ye, Q. W. Zhao, L. Y. Mai, Y. F. Huang, S. Q. Wang, J. Chen, B. Li, B. R. He, Y. Zhang, J. J. Zou, B. Z. Tang, X. H. Pan, L. Zheng. Adv. Sci. 2020, 7, 1902760.

[14] Y. Li, Z. Zhao, J. J. Zhang, R. T. K. Kwok, S. Xie, R. B. Tang, Y. X. Jia, J. C. Yang, L. Wang, J. W. Y. Lam, W. F. Zheng, X. Y. Jiang, B. Z. Tang, Adv. Funct. Mater. 2018, 1804632.

[15] C. Dias, J. P. Pais, R. Nunes, M. T. Blazquez, J. T. Marques, A. F. Almeida, P. Serra, N. M. Xavier, D. Vila-Vicosa, M. Machuqueiro, A. S. Viana, A. Martins, M. S. Santos, A. Pelerito, R. Dias, R. Tenreiro, M. C. Oliveira, M. Contino, N. A. Colabufo, R. F. M. de Almeida, A. P. Rauter, Nat. Commun. 2018, 9, 10.1038/s41467-018-06488-4.

[16] C. L. Zhu, Q. Yang, F. T. Lv, L. B. Liu, S. Wang, Adv. Mater. 2013, 25,1203.

[17] Y. Liu, S.Y. Ding, R. Dietrich, E. Märtlbauer, K. Zhu, Angew. Chem. Int. Ed. 2017, $56,1$.

[18] National Committee for Clinical Laboratory Standards. Methods for determining bactericidal activity of antimicrobial agents, M26-A, 1999.

[19] X. Y. Zheng, D. Wang, W. H. Xu, S. Q. Cao, Q. Peng, B. Z. Tang, Mater. Horiz. 2019, 6, 2016. 
[20] X. J. Shi, H. P. S. Simon, J. H. C. Chau, Y. Li, Z. Y. Liu, R. T. K. Kwok, J. K. Liu, P. H. Xiao, J. J. Zhang, B. Liu, J. W. Y. Lam, B. Z. Tang. Small Methods 2020, 2000046.

[21] A. M. Cuervo, Trends Cell Biol. 2004, 14, 70.

[22] a) A. Kuma, M. Matsui, N. Mizushima, Autophagy 2007, 3, 323. b) H. Liu, S. F. Wei, L. Zhang, C. X. Yuan, Y. Y. Duan, Q. W. Wang, Pathol. Oncol. Res. 2019, 25, 1487.

[23] N. Bravo-Santano, J. K. Ellis, L. M. Mateos, Y. Calle, H. C. Keun, V. Behrends, M. Letek, Msphere 2018, 3, e00374.

[24] M. B. Mestre, C. M. Fader, C. Solar, M. I. Colombo, Autophagy 2010, 6, 110.

[25] T. Feldkamp, A. Kribben, J. M. Weinberg, Am. J. Physiol. Renal. Physiol. 2015, 288, F1092.

[26] Q. Yang, M. Li, O. B. Spiller, D. O. Andrey, P. Hinchliffe, H. Li, C. Maclean, P. Niumsup, L. Powell, M. Pritchard, A. Papkou, Y. B. Shen, E. Portal, K. Sands, J. Spencer, U. Tansawai, D. Thomas, S. L. Wang, Y. Wang, J. Z. Shen, T. Walsh, Nat. Commun. 2017, 8, 10.1038/s41467-017-02149-0.

[27] a) M. A. Bhat, M. Imran, S. A. Khan, N. Siddiqui, Indian J. Pharm. Sci. 2005, 67, 151. b) C. T. Supuran, A. Casini, A. Mastrolorenzo, A. Scozzafava, Mini-Rev. Med. Chem. 2004, 4, 625.

[28] E. G. Zhao, Y. L. Chen, H. Wang, S. J. Chen, J. W. Y. Lam, C. W. T. Leung, Y. N. Hong, B. Z. ACS Appl. Mater. Inter. 2015, 7, 7180.

[29] J. J. Chen, M. Gao, L, Wang, S. W. Li, J. C. He, A. J. Qin, L. Ren, Y. J. Wang, B. Z. Tang. ACS Appl. Mater. Inter. 2018, 10, 11436.

[30] S. H. E. Kaufmann, A. Dorhoi, R. S. Hotchkiss, R. Bartenschlager, Nat. Rev. Drug Discov. 2018, 17, 35 .

[31] S. Tiberi, N. du Plessis, G. Walzl, M. J. Vjecha, M. Rao, F. Ntoumi, S. Mfinanga, N. Kapata, P. Mwaba, T. D. McHugh, G. Ippolito, G. B. Migliori, M. J. Maeurer, A. Zumla. Lancet Infect. Dis. 2018, 18, e183. 
[32] CLSI. Performance Standards for Antimicrobial Susceptibility Testing, 28th ed.; CLSI

Supplement M100; Clinical and Laboratory Standards Institute: Wayne, PA, USA, 2018.

[33] W. L.Garner, Plast. Reconstr. Surg. 1998, 102, 135.

[34] S. Heeb, M. P. Fletcher, S. R. Chhabra, S. P. Diggle, P. William, M. Camara. FEMS Microbiol. Rev. 2011, 35, 247.

[35] H. Hamamoto, M. Urai, K. Ishii, J. Yasukawa, A. Paudel, M. Murai, T. Kaji, T. Kuranaga, K. Hamase, T. Katsu, J. Su, T. Adachi, R. Uchida, H.Tomoda, M. Yamada, M. Souma, H. Kurihara, M. Inoue, K. Sekimizu. Nat. Chem. Biol. 2015, 11, 127.

[36] P. Vergidis, M. S. Rouse, G. Euba, M. J. Karau, S. M. Schmidt, J. N. Mandrekar, J. M. Steckelberg, R. Patel, Antimicrob. Agents Ch. 2011, 55, 1182. 